\title{
Study on the Expression of Osteopontin Coding Gene in Ovarian Cancer
}

\section{Lihong Bao}

Medical College, Inner Mongolia University for the Nationalities, Tongliao, Inner Mongolia, 028000, China

baolihong71@163.com

Keywords: Osteopontin (OPN), Ovarian Cancer, Coding Gene

\begin{abstract}
Osteopontin (OPN), as a malignant transformation related protein phosphorylation was firstly completed the extraction by Senger in 1979. With the progress of research on osteopontin (OPN), people found out that there was little amount in the normal tissues that was showed with low expression, however, in a variety of malignant tumors, the amount of OPN was over expressed. In this paper, it takes the structure and function of osteopontin as the breakthrough point, exploring the expression and the function of osteopontin (OPN) in ovarian cancer.
\end{abstract}

\section{Introduction}

Ovarian cancer is one of the common malignant tumors of the female reproductive organs, the incidence rate is inferior to cervical cancer and carcinoma of endometrium, which can rank the third place among the tumors, because there are no symptoms in the early clinical stage of ovarian cancer, and the founded tumors by carrying on operation of laparotomy confined to only $30 \%$ of the ovaries. When it was founded, most of them had the diffusion and transferred. Therefore, the mortality rate of ovarian cancer accounted for the first place among all types of gynecological tumors. Osteopontin (OPN) is a kind of secretory calcium binding protein phosphorylated glycoprotein, OPN is associated with gastric cancer, nasopharyngeal carcinoma, lung cancer, while relationship with ovarian cancer was rarely reported in China.

\section{The Structure of Osteopontin}

Osteopontin (OPN) as a malignant transformation of the phosphorylation of protein extraction was earliest extracted by Senger in 1979. In1987 under the control of protein kinase C, it was cloned as a tumor promoter induced genes. Compared with non- transformed cells, it can have high expression in many transformed cells, which also can have the over-expression in many tumors.

The human beings' OPN gene is located in $4 q 13$, which is composed of seven exons and six introns. OPN is not only an adhesive protein, but also is a cytokine, which is a kind of secretory calcium binding phosphorylated glycoprotein with multiple functions, and its relative molecular mass is $41500 \mathrm{kd}$. The Ser residue of this acidic protein can be phosphorylated, which can carry with negative charge, at the same time, it is lack of two level structure that can be extended. Encoding the sequence of the single gene is highly conserved in vertebrates, which can be the same with many extracellular matrix proteins, with cell adhesion sequence, namely, the sequence of (Arg-Gly-Asp, RGD). Therefore, it can have regulation on the transcription of the malignant cells.

\section{The Function of Osteopontin}

OPN has many biological functions, which can play roles that may be not exactly the same in different tissues. In the bone matrix, OPN is mainly involved in the resorption and formation of bone; in the tissue of inflammation, it mainly through the effect of cell signal to have transduction, so as to promote the adhesion and migration of inflammatory cells.

Xie and other people use antisense gene therapy model to show that OPN can promote the aggregation of macrophage. In addition, as for the aspect of the damages of organs, OPN can play the role of being protective agent, it can increase the tolerance ability of cells to the acute ischemia; 
restrain the biological synthesis of nitric oxide synthase and nitric oxide; enhance the cell tolerance to hypoxia; reduce the period of cell apoptosis. Parathyroid hormone, vitamin D, calcium, phosphate and so on can promote the expression of OPN both in vitro and vivo; at the same time, estrogen, the inhibitors of angiotensin converting enzyme, angiotensin prime II receptor as well as antagonistic agents can inhibit the expression of OPN. Osteopontin receptor family can be including integrin receptor and $\mathrm{CD}_{44}$ receptor. And the one that can be combined with $\mathrm{C}$ terminus belongs to the $\mathrm{CD}_{44}$ family, among them, the combination of OPN-CD $\mathrm{C}_{44} \mathrm{~V}_{6}$ can be carried out by amino acid polycondensation, and the expression of $\mathrm{CD}_{44} \mathrm{~V}_{6}$ can be the necessary condition for the invasion of tumors.

\section{The Expression and Function of Osteopontin in Ovarian Cancer}

There is a thrombin interaction site in OPN molecule, which can carry out the hydrolization of OPN, after the hydrolysis of OPN molecule and the adhesion of cells will be decreased, Xuan and other people believed that the shear of thrombin on OPN may be a kind of natural physiological regulation of body with functions. In addition, the structure of OPN also can be including a signal peptide sequence, from 7 to 10, a consecutive ASP sequence, and the combined site of calcium, the binding domains of heparin, as well as other multiple functional domains. The unique and complex molecular structure of OPN can make it play a very important role in the promotion of the development process of tumors. OPN can promote the growth and proliferation of tumor cells, inducing the formation of neovascularization, which also can enhance tumor cells to have adhesion and migration through a variety of ways, so as to participate in the invasion and metastasis of tumors.

A large number of studies have showed that OPN had close relationship with the occurrence, development, metastasis and prognosis of a lot of malignant tumors. As for the expression of OPN in ovarian cancer, there are some studies both at home and abroad. Kim and other staff studied the expression of OPN in normal ovarian tissues, ovarian borderline tumors and malignant ovarian tumor tissue by adopting the immunohistochemical method. The results showed that there was no expression of OPN in normal ovarian tissues, however, the expression positive rate in malignant ovarian tissue was significantly higher than that of the positive rate in borderline tumors, which was statistically significant not only in the occurrence of ovarian cancer, but also in the process of development with its great significance.

Rosen and other researching staff found out that there was no expression of OPN in the normal ovarian cells, but in ovarian cystadenoma, borderline ovarian tumor and serous ovarian cancer, the expression of them was increased gradually, which also can indicate that there is a close relationship between the occurrence and development of ovarian cancer with OPN. 
Type of organization

Normal ovarian tissue

Benign ovarian tumor
The expression of OPN

The rate of expression

$\begin{array}{lllll}\text { Epithelial ovarian cancer } & 60 & 15 & 45 & 75 \%\end{array}$

Note: the comparison of the positive expression rate of OPN among the groups:

(1) normal group-malignant group: $\chi^{2}=34.286 \quad \mathrm{P}=0.000$;

(2) benign group-malignant group: $\chi^{2}=34.286 \quad \mathrm{P}=0.000$;

(3) normal group- benign group: $\chi^{2}=0.360 \quad \mathrm{P}=0.548$.

Table 2 The Expression of Protein $\alpha_{v} \beta_{3}$ in Different Ovarian Tissues

Type of organization

Normal ovarian tissue
The expression of $\alpha_{\mathrm{v}} \beta_{3}$
The rate of expression

\begin{tabular}{ccccc} 
Normal ovarian tissue & 20 & 20 & 0 & $0 \%$ \\
Benign ovarian tumor & 20 & 19 & 1 & $5 \%$ \\
Epithelial ovarian cancer & 60 & 22 & 38 & $63.3 \%$ \\
\hline
\end{tabular}

Note: the comparison of the positive expression rate of $\alpha v \beta 3$ among the groups:

(1)normal group-malignant group: $\chi^{2}=24.127 \quad \mathrm{P}=0.000$;

(2) benign group-malignant group: $\chi^{2}=20.429 \quad \mathrm{P}=0.000$; (3) normal group-benign group: $\chi^{2}=1.026 \quad \mathrm{P}=0.311$. 
Table3 The Correlation of the Expression of OPN with $\alpha v \beta 3$ in Ovarian Cancer

\begin{tabular}{cccc} 
Group & OPN(+) & OPN(-) & Total \\
\hline$\alpha_{\mathrm{v}} \beta_{3}(+)$ & 36 & 2 & 38 \\
$\alpha_{\mathrm{v}} \beta_{3}(-)$ & 9 & 13 & 22 \\
Total & 45 & 15 & 60 \\
\hline
\end{tabular}

Note: Group of OPN-Group of $\alpha v \beta 3: \chi^{2}=21.531 \quad \mathrm{P}=0.000$.

\section{Conclusion}

This study adopts the method of immunohistochemistry, so as to have detection on the expression of OPN gene in benign and malignant ovarian tumors. The results showed that the positive expression rate of OPN gene in malignant tumor was significantly higher, which was significantly different from its expression in benign tumor. Since it had statistically significance, thus, OPN may have close relationship with the occurrence and development of malignant ovarian tumor. This result is consistent with the result of the expression of OPN in ovarian cancer made by Kim and other staff.

Through the circumstances of expression and relationship between OPN gene in benign and malignant ovarian tumors in clinical parameters, it can make analysis and do research. Thus it can find out that OPN gene in lymph node metastasis group is significantly higher than that without lymph node metastasis. While the expression rate in clinical stage III and IV can be higher than the rate of patients in stage I and stage II, while the difference has statistical significance. At the same time, the result is consistent with the current results in the literature or some other reports. Therefore, OPN can play an very important role in the occurrence and development of ovarian cancer, the recurrence and metastasis of ovarian cancer. And there was no significant difference for the expression of OPN gene in different ages or different pathological types of ovarian cancer.It means that there is little concern between the function of OPN to promote the occurrence and development of the tumors, which can be consistent with the researches that we had made in recent years, namely, OPN can promote the occurrence and development of tumors.

\section{References}

[1]Thalmann GN, Sikes Ra, Devoll RE, Kiefer JA, et al.1999. Osteopontin: possible role in prostate cancer progression. Clin Cancer Res. vol.5, pp2271-2277.

[2]Scott JA,Weir ML,Wilson SM,et al. Osteopontin inhibits inducible nitric oxide synthase activity in rat vascular tissue. Am J Phuiol, 1998, 275:2258-2265.

[3]Haynes BF,Denning SM, Subger KH, et al. 1989. Ontogeny of T-cell precurors:a model for the initial stages of human T-cell development.Immu,Today. vol.103, pp87-91.

[4]S Goodison,V Urquidi, D Tarin. 1999. CD $_{44}$ cell adhesion molecules.Clin Pathol: Mol Pathol. vol.52, pp189-196.

[5]Kim JH, Skates SJ, Uede T, et al. 2002. Osteopontin as a potential diagnostic biomarker for ovarian cancer. JAMA. vol.287, pp1671-1679. 